Quim. Nova, Vol. 35, No. 10, 2072-2075, 2012

\title{
REAÇÕES ÁCIDO-BASE: CONCEITO, REPRESENTAÇÃO E GENERALIZAÇÃO A PARTIR DAS ENERGIAS ENVOLVIDAS NAS TRANSFORMAÇÕES
}

\author{
Iêda Aparecida Pastre, Lídia Maria de Almeida Plicas e Vera Aparecida de Oliveira Tiera* \\ Departamento de Química e Ciências Ambientais, Instituto de Biociências, Letras e Ciências Exatas, Universidade Estadual Paulista \\ "Julio de Mesquita Filho", Rua Cristóvão Colombo, 2265, 15054-000 São José do Rio Preto - SP, Brasil \\ Juliana Vieira Custódio e Silvia Maria Leite Agostinho \\ Departamento de Química Fundamental, Instituto de Química, Universidade de São Paulo, CP 26077, 05513-970 São Paulo - SP, \\ Brasil
}

Recebido em 23/2/12; aceito em 13/6/12; publicado na web em 4/9/12

\begin{abstract}
ACID-BASE REACTIONS: CONCEPT, REPRESENTATION AND GENERALIZATION FROM THE ENERGY INVOLVED IN TRANSFORMATIONS. Undergraduate students on the first year of Chemistry Courses are unfamiliar with the representation of acid-base reactions using the ionic equation $\mathrm{H}^{+}+\mathrm{OH}^{-} \rightarrow \mathrm{H}_{2} \mathrm{O}$. A chemistry class was proposed about acid-base reactions using theory and experimental evaluation of neutralization heat to discuss the energy involved when water is formed from $\mathrm{H}^{+}$and $\mathrm{OH}^{-}$ions. The experiment is suggested using different strong acids and strong base pairs. The presentation of the theme within a chemistry class for high school teachers increased the number of individuals that saw the acid-base reaction from this perspective.
\end{abstract}

Keywords: acid-base reactions; neutralization heat; ionic equation.

\section{INTRODUÇÃO}

Uma das dificuldades encontradas pelos alunos ingressantes em cursos de graduação de Química consiste no conhecimento da representação mais apropriada para descrever as transformações químicas. As reações ácido-base conforme a teoria de Arrhenius podem ilustrar essa dificuldade: ${ }^{1}$ verifica-se que a maioria dos estudantes ingressantes sabe dar como exemplo a reação entre hidróxido de sódio e ácido clorídrico, e representam esta transformação empregando a equação molecular, isto é $\mathrm{NaOH}(\mathrm{aq})+\mathrm{HCl}(\mathrm{aq}) \mathrm{NaCl}(\mathrm{aq})+\mathrm{H}_{2} \mathrm{O}(\mathrm{l})$. Verificamse dois problemas na formação destes estudantes: a visão restrita e muito particular de um processo ácido-base e o desconhecimento da representação envolvendo íons. Esta constatação se baseia em uma avaliação, realizada pelos autores, com alunos ingressantes em cursos de Graduação em Química, Licenciatura e Bacharelado. A avaliação foi feita dentro da disciplina de Química Geral, no primeiro mês de aula no $1^{\circ}$ ano, em uma universidade pública e em uma faculdade particular. O tema escolhido foi, a representação das transformações químicas ácido-base na forma de equações químicas. A avaliação foi baseada no seguinte texto:

"O hidróxido de sódio, $\mathrm{NaOH}$, é uma base e o $\mathrm{HNO}_{3}$ é o ácido nítrico. a) Escreva a equação representativa da reação

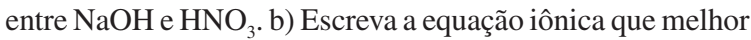
representa esta reação. c) Dê outro exemplo de reação ácido base, equacionando na forma que você achar mais adequada. d) Qual a equação genérica, na forma iônica, que representa a reação ácido-base em meio aquoso?"

As respostas ao questionário aplicado foram analisadas e as percentagens de acertos listadas na Tabela 1 .

Observa-se que a alta porcentagem de acertos nas questões a) e c) se devem ao fato de que foram consideradas certas as equações representadas na forma molecular. Quando solicitada a equação na forma iônica (questão b) verificou-se um decréscimo considerável

*e-mail: verapoli@ibilce.unesp.br
Tabela 1. Percentual de acertos entre os alunos ingressantes

\begin{tabular}{lccccc}
\hline \multirow{2}{*}{ Grupos analisados } & \multirow{2}{*}{$\begin{array}{c}\mathrm{N}^{\circ} \mathrm{de} \\
\text { alunos }\end{array}$} & \multicolumn{4}{c}{ Questões } \\
\cline { 3 - 6 } & 151 & $94 \%$ & $\mathrm{~b}$ & $\mathrm{c}$ & $\mathrm{d}$ \\
\hline Universidade pública & 85 & $58 \%$ & $28 \%$ & $51 \%$ & $30 \%$ \\
Faculdade particular & & & &
\end{tabular}

na porcentagem de acertos e grande parte das respostas consideradas corretas apresentavam íons, que não participam da transformação química, dos dois lados da equação. Este fato justifica a dificuldade encontrada na descrição geral da reação ácido-base solicitada no item (d). Os alunos mostraram uma deficiência em perceber que a equação química genérica da reação entre um ácido e uma base em meio aquoso leva à formação de água a partir dos íons $\mathrm{H}_{3} \mathrm{O}^{+}$e/ou $\mathrm{H}^{+}$e $\mathrm{OH}^{-}$solvatados (questão d). Verifica-se também que os alunos ingressantes nas universidades públicas apresentam um melhor percentual de acertos em relação àqueles que ingressam na particular, refletindo, na visão dos autores, diferenças resultantes de um maior rigor no exame vestibular das primeiras.

Um estudo realizado por Fernandez e colaboradores sobre os conceitos de química dos ingressantes em cursos de graduação do Instituto de Química da Universidade de São Paulo mostrou resultado semelhante. ${ }^{2}$ Quando os autores apresentaram uma questão envolvendo a variação de entalpia $(\Delta \mathrm{H})$ nas reações em meio aquoso entre $\mathrm{HCl}$ e $\mathrm{NaOH}$ e entre $\mathrm{HNO}_{3}$ e $\mathrm{KOH}$, apenas $26 \%$ dos ingressantes escolheram a alternativa certa, de que o valor de $\Delta \mathrm{H}$ é o mesmo nos dois processos porque "a reação envolvida é sempre de íons $\mathrm{H}^{+} \mathrm{e}$ íons $\mathrm{OH}^{-}$, sem a participação de outros íons". Neste trabalho os autores concordam com Nery e colaboradores: " "não é hábito dos alunos pensar nas reações sob a forma iônica". Drechsler e colaboradores mostram em seu trabalho que a equação preferida pelos estudantes para descrever a reação entre $\mathrm{HCl}$ e $\mathrm{NaOH}$ foi a seguinte: ${ }^{4} \mathrm{Na}^{+}+\mathrm{Cl}^{-}+$ $\mathrm{H}^{+}+\mathrm{OH}^{-} \AA \mathrm{NaCl}+\mathrm{H}_{2} \mathrm{O}$ em lugar de $\mathrm{H}^{+}+\mathrm{OH}^{-}{ }^{\circledR} \mathrm{H}_{2} \mathrm{O}$.

Em vista desta constatação, o tema foi inserido em um curso de capacitação (extensão universitária) para professores do ensino médio público da região de São José do Rio Preto. Os cursos de 
aprimoramento dos professores de Química do Ensino Médio, da região de São José do Rio Preto, atenderam uma clientela de aproximadamente 150 profissionais, no período de 1999 a 2010, onde foi avaliado o perfil profissional dos mesmos. Os dados coletados no período, por Pastre e colaboradores, ${ }^{5}$ mostram que a maioria $(81 \%)$ são oriundos de Faculdades privadas, Figura 1a. Quanto à formação profissional, a maioria dos professores de Química que atuam no Ensino Médio possui formação em áreas afins como Licenciatura em Ciências com Habilitação em Química e Ciências Biológicas, sendo que apenas $18 \%$ apresentam formação específica em Licenciatura em Química, Figura 1b. O prognóstico realizado evidenciou que embora habilitados em Química, a precária formação desses profissionais os deixava inseguros para a montagem e realização de aulas experimentais, mesmo a escola dispondo de recursos necessários para o desenvolvimento dessas aulas. Ficou claro também que muitos destes profissionais não realizaram atividades de laboratório nos seus cursos de formação.

(a)

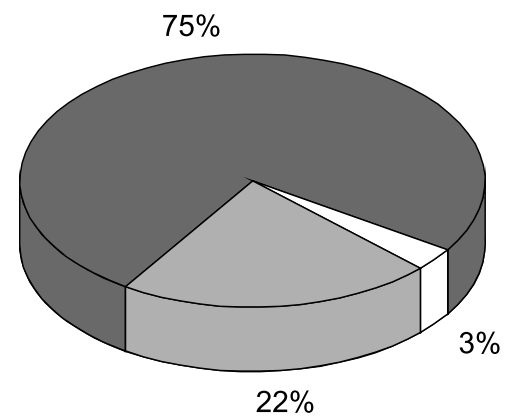

Outras áreas

Licenciatura em Química

Licenciatura em Ciências com Habilitação em Química

(b)

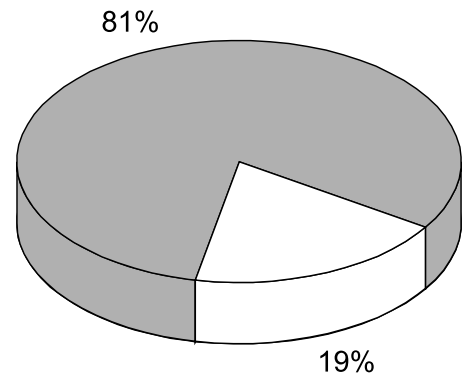

Universidades Públicas

Faculdades Particulares

Figura 1. (a) Qualificação do professor de química da região de São José do Rio Preto - dados coletados no período de 2001 a 2007. (b) Porcentagem de professores oriundos de instituições públicas e privadas

O curso realizado em 2010, no Departamento de Química e Ciências Ambientais do Instituto de Biociências Letras e Ciências Exatas (IBILCE/UNESP) em São José do Rio Preto, foi oferecido aos professores, que ministram a disciplina de química, com o objetivo de avaliar, discutir e ampliar o conhecimento da representação das transformações químicas na forma de equações químicas. Na parte referente às reações ácido-base o curso foi precedido e sucedido pela aplicação do mesmo questionário descrito anteriormente. É importante salientar que o tema reações ácido-base foi precedido de aulas teóricas envolvendo os seguintes conceitos: mol, água como solvente, números de mol em $1 \mathrm{~L}$ de solvente, íons, solvatação, energias envolvidas nas transformações.

Além da avaliação conceitual por meio do questionário, a aula ácido-base teve como objetivos: 1) propor um experimento de termoquímica em que fosse determinado o calor envolvido na reação de neutralização de um ácido forte por uma base forte, empregando diferentes pares ácido-base; 2) comparar os resultados obtidos pelos professores, levando em conta os desvios das medidas experimentais, as operações com desvios e a propagação de erros; 3 ) levar os professores, a partir do experimento realizado e dos resultados obtidos, à generalização das reações ácido-base, como sendo a formação de água a partir de íons $\mathrm{H}^{+}(\mathrm{aq})$ e $\mathrm{OH}^{-}(\mathrm{aq})$ e, 4) mostrar, a partir desses resultados, a importância da representação das reações envolvidas na forma de equação iônica.

Acredita-se que uma discussão teórica sobre as energias envolvidas nas transformações ácido-base acrescidas de um experimento de termoquímica possa levar os alunos a uma generalização empregando a equação $\mathrm{H}^{+}(\mathrm{aq})+\mathrm{OH}^{-}(\mathrm{aq}) \rightarrow \mathrm{H}_{2} \mathrm{O}$.

É importante salientar que, embora no passado, experimentos termoquímicos tenham sido propostos por vários autores ${ }^{6-8}$ com o objetivo de determinar o calor de neutralização, nos últimos anos os experimentos envolvendo ácidos e bases estão mais voltados para medidas de $\mathrm{pH}$ e titulações volumétricas. ${ }^{9} \mathrm{O}$ calor de neutralização vem sendo apresentado como um dado fornecido na determinação da capacidade calorífica do calorímetro, ${ }^{10}$ servindo de ponto de partida para determinação da variação de entalpia de processos mais complexos ou, mais recentemente, como um dado fornecido com o objetivo de padronizar o calorímetro. ${ }^{8}$

\section{PROPOSTA DE EXPERIMENTO}

Um experimento que envolve diferentes combinações ácido-base pode ser aplicado para a determinação do calor de neutralização $\left(Q_{N}\right)$ como uma forma de sanar as dificuldades envolvendo conceitos químicos referentes à representação de uma reação ácido-base. Uma variante deste experimento se encontra na literatura, ${ }^{2}$ onde o calor de neutralização é conhecido e se emprega a reação ácido-base com o objetivo de determinar a capacidade calorífica do calorímetro.

Para a realização desta proposta foi interessante dividir a turma de laboratório em 4 grupos, cada qual empregando um par ácido-base diferente. Foram escolhidos como ácidos fortes, soluções aquosas de ácido clorídrico e ácido nítrico e, como bases fortes, soluções aquosas de hidróxido de sódio e hidróxido de potássio.

\section{Material necessário}

Termômetros tendo como menor divisão $1^{\circ} \mathrm{C}$ (precisão de 0,5 ${ }^{\circ} \mathrm{C}$ ), calorímetro simples; soluções aquosas 1,00 mol L-1 de $\mathrm{HCl}$, $\mathrm{HNO}_{3}, \mathrm{NaOH}$ e $\mathrm{KOH}$; béquer de $100,0 \mathrm{~mL}$ e pipetas de $25,00 \mathrm{~mL}$. O calorímetro deve ser basicamente constituído de um béquer inserido em recipiente cilíndrico de isopor, no qual se encaixa perfeitamente, como ilustrado na Figura 2. A tampa do recipiente, também de isopor, contém orifícios para adaptação do termômetro e do agitador manual.

\section{Cálculo da capacidade calorífica do calorímetro}

Colocar, em béquer de $100 \mathrm{~mL}, 25,0 \mathrm{~mL}$ de água fria, à temperatura ambiente, $\mathrm{T}_{1}$. Adicionar $25,0 \mathrm{~mL}$ de água quente, a uma temperatura $\mathrm{T}_{2}$. A capacidade calorífica $\mathrm{C}$, definida como a energia necessária para elevar de um grau a massa do calorímetro e acessórios, ${ }^{1,11,12}$ pode ser obtida a partir das Equações 1 a 4 .

$$
\begin{gathered}
\mathrm{Q}_{\mathrm{Q}}=\mathrm{Q}_{\mathrm{F}}+\mathrm{Q}_{\mathrm{C}} \\
\mathrm{Q}_{\mathrm{F}}=\mathrm{C}_{\mathrm{H} 2 \mathrm{O}} \mathrm{m}_{\mathrm{H} 2 \mathrm{O}}\left(\mathrm{T}_{3}-\mathrm{T}_{1}\right) \\
\mathrm{Q}_{\mathrm{Q}}=\mathrm{C}_{\mathrm{H} 2 \mathrm{O}} \mathrm{m}_{\mathrm{H} 2 \mathrm{O}}\left(\mathrm{T}_{2}-\mathrm{T}_{3}\right) \\
\mathrm{Q}_{\mathrm{C}}=\mathrm{C}\left(\mathrm{T}_{3}-\mathrm{T}_{1}\right)
\end{gathered}
$$




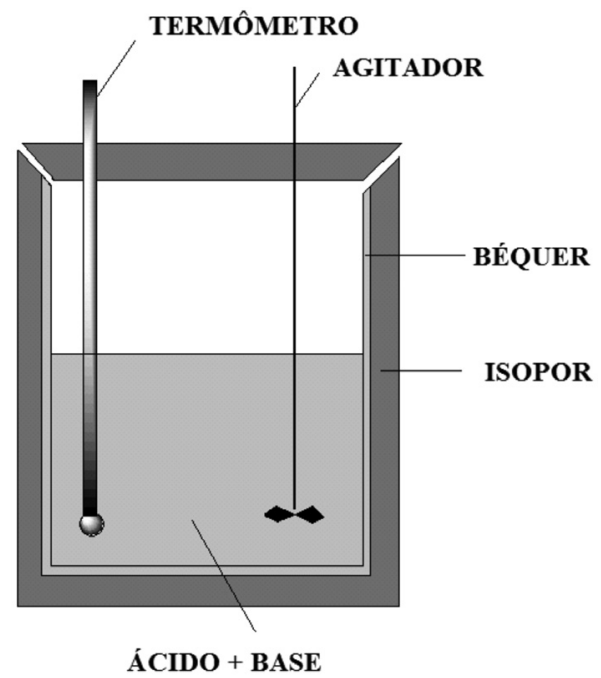

Figura 2. Esquema do calorímetro utilizado nos experimentos

onde: $\mathrm{Q}_{\mathrm{Q}}$ - calor cedido pela água quente; $\mathrm{Q}_{\mathrm{F}}$ - calor recebido pela água fria; $\mathrm{Q}_{\mathrm{C}}$ - calor recebido pelo calorímetro e acessórios; $\mathrm{C}_{\mathrm{H} 2 \mathrm{O}}=$ calor específico da água; $\mathrm{m}_{\mathrm{H} 2 \mathrm{O}}=$ massa de água; $\mathrm{T}_{1}=$ temperatura inicial da água fria e do calorímetro; $\mathrm{T}_{2}=$ temperatura inicial da água quente; $\mathrm{T}_{3}=$ temperatura final e, $\mathrm{C}=$ capacidade calorífica do calorímetro e acessórios.

No cálculo da capacidade calorífica devem ser levados em conta os desvios das medidas e as operações com esses desvios.

\section{Determinação do calor de neutralização $\left(Q_{N}\right)$}

Após a determinação de $\mathrm{C}$ procede-se à determinação de $\mathrm{Q}_{\mathrm{N}}$. São adicionados 25,0 $\mathrm{mL}$ da solução de $1,00 \mathrm{~mol} \mathrm{~L}^{-1}$ de $\mathrm{HCl}$ (ou $\mathrm{HNO}_{3}$ ) ao calorímetro, anotando-se a temperatura da solução. Em seguida, igual volume de uma solução $1,00 \mathrm{~mol} \mathrm{~L}^{-1}$ de $\mathrm{NaOH}$ (ou KOH) é medido e sua temperatura anotada. Esta solução é adicionada ao calorímetro, contendo o ácido, provido de agitador mecânico e termômetro. As temperaturas, inicial e final, depois de feita a mistura do ácido e da base, são anotadas e, a partir destes resultados, é calculado o calor de neutralização por mol de água formada para cada par ácido-base. $\mathrm{O}$ calor de neutralização por mol de água formada, $\mathrm{Q}_{\mathrm{N}}$, é obtido a partir da Equação 5:

$$
\mathrm{q}_{\mathrm{n}}=\mathrm{q}_{\mathrm{s}}+\mathrm{q}_{\mathrm{c}}
$$

onde: $\mathrm{q}_{\mathrm{n}}$ - calor envolvido na neutralização de $\mathrm{n}$ mols de água formados a partir de $\mathrm{n}$ mols de ácido e base misturados; $\mathrm{q}_{\mathrm{s}}$ - calor recebido pela solução, dado por $\mathrm{q}_{\mathrm{s}}=\mathrm{C}_{\mathrm{s}} \mathrm{m}_{\mathrm{s}}\left(\mathrm{T}_{\mathrm{f}}-\mathrm{T}_{\mathrm{i}}\right)$, sendo $\mathrm{C}_{\mathrm{s}}$ o calor específico da solução e $\mathrm{T}_{\mathrm{i}}$ e $\mathrm{T}_{\mathrm{f}}$ a temperatura inicial e final, respectivamente; $\mathrm{q}_{\mathrm{c}}$ - calor recebido pelo calorímetro, dado por $\mathrm{q}_{\mathrm{c}}=\mathrm{C}\left(\mathrm{T}_{\mathrm{f}}-\mathrm{T}_{\mathrm{i}}\right)$.

Conhecido o valor de $\mathrm{q}_{\mathrm{n}}$ calcula-se $\mathrm{Q}_{\mathrm{N}}$ pela relação:

$$
\mathrm{Q}_{\mathrm{N}}=\mathrm{q}_{\mathrm{n}} / \mathrm{n}
$$

\section{RESULTADOS OBTIDOS EM UM CURSO DE CAPACITAÇÃO PARA PROFESSORES DO ENSINO MÉDIO}

Na Tabela 2 são apresentados os resultados obtidos na aplicação do questionário aos professores. Observa-se, de maneira semelhante ao que ocorreu com os alunos, a queda no percentual de acertos na questão (b), em que os professores teriam que representar a transformação química na forma de equação iônica. Grande parte das respostas consideradas corretas apresentava íons que não participavam da reação química, dos dois lados da equação, como descrito a seguir:

$\mathrm{Na}^{+}(\mathrm{aq})+\mathrm{OH}^{-}(\mathrm{aq})+\mathrm{H}^{+}(\mathrm{aq})+\mathrm{NO}_{3}^{-}(\mathrm{aq}) \rightarrow \mathrm{H}_{2} \mathrm{O}+\mathrm{Na}^{+}(\mathrm{aq})+\mathrm{NO}_{3}^{-}(\mathrm{aq})$

Neste exemplo, os referidos íons seriam $\mathrm{Na}^{+}(\mathrm{aq})$ e $\mathrm{NO}_{3}^{-}(\mathrm{aq})$.

Tabela 2. Percentual de acertos na aplicação do questionário no início do curso

\begin{tabular}{lccccc}
\hline \multirow{2}{*}{ Grupo analisado } & \multirow{2}{*}{$\mathrm{N}^{\circ}$ total } & \multicolumn{5}{c}{ Questões } \\
\cline { 3 - 6 } & & $\mathrm{a}$ & $\mathrm{b}$ & $\mathrm{c}$ & $\mathrm{d}$ \\
\hline Professores & 24 & $96 \%$ & $42 \%$ & $88 \%$ & $8 \%$ \\
\hline
\end{tabular}

Os professores de ensino médio ainda apresentaram baixo percentual de acerto, mesmo quando estimulados, após discussão do tema dentro do curso de extensão universitária, com aulas teóricas e práticas.

Também se observa, na Tabela 2, que apenas $8 \%$ dos professores souberam generalizar a neutralização representada pela equação $\mathrm{H}^{+}(\mathrm{aq})+\mathrm{OH}^{-}(\mathrm{aq}) \rightarrow \mathrm{H}_{2} \mathrm{O}(\mathrm{l})$ e responder à questão $(\mathrm{d})$.

\section{Avaliação final dos professores}

Os valores médios de $\mathrm{Q}_{\mathrm{N}}$ e os percentuais de acerto para a questão "Qual a equação genérica que representa a reação de neutralização envolvendo um ácido forte e uma base forte?" se encontram na Tabela 3. Considerou-se como resposta certa a equação:

$$
\mathrm{H}^{+}(\mathrm{aq})+\mathrm{OH}^{-}(\mathrm{aq}) \rightarrow \mathrm{H}_{2} \mathrm{O}(\mathrm{l})
$$

Tabela 3. Valores de $\mathrm{Q}_{\mathrm{N}}$ obtidos e percentual de acertos à questão (d)

\begin{tabular}{ccc}
\hline Grupo analisado & $\mathrm{QN}\left(\mathrm{kJ} \mathrm{mol}^{-1}\right)$ & $\%$ Acerto (questão d) \\
\hline Professores & $60 \pm 10$ & 19 \\
\hline
\end{tabular}

No experimento proposto neste trabalho foi sugerido um termômetro com precisão de $0,5^{\circ} \mathrm{C}$. No caso dos professores foi calculado o desvio, no valor de QN, e este se mostrou elevado, em virtude de se empregar termômetro com esta precisão. Um valor médio igual a $53 \pm 3 \mathrm{~kJ} \mathrm{~mol}^{-1}$ foi encontrado pelos autores deste trabalho, empregando termômetro com precisão de $0,05^{\circ} \mathrm{C}$. Estes resultados quando comparados com a literatura mostram uma boa concordância: Papee e colaboradores obtiveram o valor igual a 56,5 $\pm 0,2 \mathrm{~kJ} \mathrm{~mol}^{-1},{ }^{13}$ e está de acordo com Mahoney e colaboradores, ${ }^{14}$ $55,84 \mathrm{~kJ} \mathrm{~mol}^{-1}$.

A experiência envolvendo o cálculo de $\mathrm{Q}_{\mathrm{N}}$ levou o mesmo grupo de professores a um número maior de acertos. O percentual se elevou de $8 \%$ (Tabela 2, questão d), para 19\% (Tabela 3). A questão foi formulada aos professores no final do curso, isto é, decorridos 3 meses da realização do experimento. $\mathrm{O}$ procedimento foi proposital: desejava-se verificar se o conceito havia sido retido por eles ao longo do curso.

\section{CONCLUSÕES}

A representação na forma de equações químicas que descrevem as reações ácido-base precisa ser melhor trabalhada no ensino médio e na graduação podendo, neste caso, ser utilizadas aulas expositivas, de laboratório e/ou demonstrativas.

O experimento proposto, envolvendo a troca de energia nas transformações estudadas, através da determinação do calor de neutralização, serve como instrumento para generalizar o conceito e a 
representação, na forma de equação iônica, que descrevem a reação de neutralização.

Tais conceitos devem ser enfatizados nas disciplinas básicas de química, em cursos de licenciatura ou de capacitação de professores de química do ensino médio.

A generalização do conceito de formação de moléculas de água a partir de íons $\mathrm{H}_{3} \mathrm{O}^{+}(\mathrm{aq})$ ou $\mathrm{H}^{+}(\mathrm{aq})$ e $\mathrm{OH}^{-}(\mathrm{aq})$ é feita, no experimento proposto, a partir de considerações energéticas. O professor poderá aplicar os conceitos em uma aula de termoquímica a partir de uma proposta de experimento ou da análise de valores tabelados de $\mathrm{Q}_{\mathrm{N}}$, de diferentes pares ácido forte-base forte.

\section{CONSIDERAÇOES FINAIS}

Esta aula experimental dá oportunidade a se discutir a importância dos desvios nas medidas e dos cálculos com desvios e de se avaliar a propagação do erro em medidas físico-químicas.

$\mathrm{O}$ emprego de termômetro com $1{ }^{\circ} \mathrm{C}$ de menor divisão (precisão de $0,5^{\circ} \mathrm{C}$ ) torna o experimento de baixo custo.

O professor pode discutir com os estudantes qual seria a diferença, em termos de energia, se fossem empregados um ácido fraco ou uma base fraca, levando em conta a contribuição energética envolvida no processo de ionização do eletrólito fraco.

Outro tema que pode ser discutido é a não escolha do ácido sulfúrico em virtude do calor de diluição que se soma ao de neutralização e por apresentar um grau de ionização inferior a 1,0 na segunda etapa de ionização.

\section{REFERÊNCIAS}

1. Chagas, A. P.; Quim. Nova 2000, 23, 126; Gama, M. S.; Afonso, J. C.; Quim. Nova 2007, 30, 232; Atkins, P.; Jones, L.; Princípios de química - questionando a vida moderna e o meio ambiente, $4^{\mathrm{a}}$ ed., Artmed: Porto Alegre, 2007.

2. Fernandez, C.; Baldinato, J. O.; Tiedemann, P.W.; Bertotti, M.; Quim. Nova 2008, 31, 1582.

3. Nery, A. L. P.; Liegel, R. M.; Fernandez, C.; Química Nova na Escola 2008, 23, 14.

4. Drechsler, M.; Scmidt, H-J.; Advancing the Chemical Sciences: Education in Chemistry 2005, 1.

5. Pastre, I. A.; Tiera, V. A. O.; Fertonani, F. L.; Caporalin, C. B.; Silva, C. R. F. Em Núcleos de Ensino da UNESP Artigos 2007; de Pinho, S. Z.; de Oliveira, J. B. B., orgs.; Cultura Acadêmica: São Paulo, 2011, p. 57.

6. Miller, J. G.; Lowell, A. I.; Lucasse, W. W.; J. Chem. Educ. 1947, 24 , 121.

7. Mills, K. V.; Guilmette, L. W.; J. Chem. Educ. 2007, 84, 326.

8. Assumpção, M. H. M. T.; Wolf, L. D.; Bonifácio, V. G.; Fatibello-Filho, O.; Eclética Química 2010, 35, 63.

9. Bertotti, M.; Quim. Nova 2011, 34, 1836.

10. Giesbrecht, E.; Experiências de química: técnicas e conceitos básicos: PEQ-Projetos de Ensino de Química, Ed. Moderna: São Paulo, 1979, p. 82.

11. Levine, I. N.; Physical Chemistry, McGraw Hill: New York, 1995.

12. Pilla, L.; Físico-Química, Livros Técnicos e Científicos: Rio de Janeiro, 1979 , p. 1.

13. Papee, H. M.; Canady, W. J.; Laidler, K. J.; Can. J. Chem. 1956, 34, 1677.

14. Mahoney, D. W.; Sweeney, J. A.; Davenport, D. A.; Ranette, R. W.; J. Chem. Educ. 1981, 58, 730. 